\title{
Caracterización química de la Fibra de Coco (Cocus nucifera L.) de México utilizando Espectroscopía de Infrarrojo (FTIR)
}

\section{Chemical characterization of Coco Fiber (Cocus nucifera L.) from Mexico using Infrared Spectroscopy (FTIR)}

\begin{abstract}
Núria E. Hernández Vidal
Estudiante de ingeniería agroindustrial. Universidad Autónoma Chapingo,Texcoco-México.

(Autor para correspondencia). nhernandezvidal@gmail.com

Vicente López Bautista

Doctor Maestro Ingeniero, Profesor investigador de tiempo completo del departamento de Ingeniería Agroindustrial, Universidad Autónoma Chapingo,

Texcoco-México vicentlop271011@gmail.com

W. D. Méndez Ordoñez

Estudiante de ingeniería agroindustrial. Universidad Autónoma Chapingo, Texcoco-México nhernandezvidal@gmail.com

S. E. Osorio Calderon

Estudiante de ingeniería agroindustrial. Universidad Autónoma Chapingo, Texcoco-México nhernandezvidal@gmail.com
\end{abstract}

\section{Resumen}

Existe un creciente interés en productos con menor impacto ambiental, esto hace que el uso de fibras naturales sea una alternativa viable. El uso de materiales de origen biológico está aumentando en un intento de reducir la cantidad de polímeros termoplásticos de fuentes no renovables. En este marco se presenta el trabajo caracterización química de la fibra de coco (Cocus nucifera L.), mediante espectroscopía de infrarrojo (FTIR) , y se muestran los espectros de las fibras de coco, de dos lugares de cultivo importantes, la región de Acapulco y San Jerónimo de Juárez, en el estado de Guerrero, México , los resultados obtenidos son comparados con otros espectros de otras fibras naturales, debido al alto contenido de lignina se le atribuyen propiedades viscoelásticas, por lo que se infiere que al ser sometidas las fibras a una carga de compresión o una fuerza de impacto, la región amorfa (lignina) funciona como amortiguador y es resiliente a los fenómenos aplicados, una característica deseable en los materiales de embalaje, esto prueba que la fibra de coco con aglomerante natural, pudiera ser una propuesta para usarse como material de relleno en embalajes, los datos corroboran un alto porcentaje de celulosa y lignina presente en las muestras estudiadas.

Palabras clave: fibra de coco; FTIR; compuestos orgánicos; fibras naturales; embalaje 


\begin{abstract}
There is a growing interest in products with less environmental impact, this makes the use of natural fibers a viable alternative. The use of materials of biological origin is increasing in an attempt to reduce the amount of thermoplastic polymers from non-renewable sources. In this framework, the work characterizes the chemical characterization of coconut fiber (Cocus nucifera L.), by means of infrared spectroscopy (FTIR), and the spectra of the fibers of coconut, from two important growing sites, the Acapulco and San Jerónimo de Juárez regions, in the state of Guerrero, Mexico, the results obtained are compared with other spectra of other natural fibers, due to the high content of lignin, properties are attributed viscoelastic, by which it is inferred that when the fibers are subjected to a compression load or an impact force, the amorphous region (lignin) functions as a buffer and is resilient to the applied phenomena, a desirable characteristic in packaging materials, this proves that the coconut fiber with natural binder, could be a proposal to be used as packing material in packaging, the data corroborate a high percentage of cellulose and lignin present in the samples studied.
\end{abstract}

Keywords: coconut fiber; FTIR; organic compounds;natural fibers; packaging

\section{Introducción}

La sociedad mundial es más consciente de los costos y riesgos de mantener una matriz energética altamente dependiente de los combustibles fósiles. Así, que el mundo está buscando fuentes de energía alternativas y renovables. La biomasa es actualmente la principal fuente de energía en muchos países en desarrollo y, por diferentes razones, sean ambientales o socioeconómicas también está creciendo en muchos países industrializados (Padilla, et al., 2016). Las políticas ambientales del gobierno han obligado a industrias como la automotriz, el embalaje y la construcción a buscar materiales ecológicos (Venkatachalam, et al., 2016). Al día de hoy existe creciente interés en productos que tengan, menor impacto ambiental, esto hace que el uso de fibras naturales sea una alternativa viable. El uso de materiales de origen biológico está aumentando en un intento de reducir la cantidad de polímeros termoplásticos de fuentes no renovables (D'Lemos, et al., 2017).

Debido a esto, se han usado diferentes fibras naturales, tales como fibras de madera (lignum), fibra de coco (cocoes), algodón (Gossypium), yute (Corchorus capsularis), sisal (Agave sisalana), curauá (Ananas lucidus), kenaf (Hibiscus cannabinus) y bambú (Bambusoideae), en diferentes aplicaciones de ingeniería. En relación a la fibra de coco alrededor de 62 millones de toneladas son cultivadas en 92 países en el mundo. Los árboles de coco o palmeras y las cáscaras de la fruta del coco son ampliamente usadas cómo fuentes de fibras. Las fibras obtenidas a partir de la cáscara del coco son generalmente llamadas fibras de la médula del coco y son usadas en una variedad de aplicaciones (Reddy \& Yang, 2015). La espectroscopía bidimensional de infrarrojo (IR 2D) ha surgido recientemente como una poderosa herramienta con aplicaciones en muchas áreas de la investigación científica. La alta resolución de tiempo inherente junto con la resolución espacial específica de enlace de la espectroscopía IR permite la caracterización directa de especies rápidamente interconversas y procesos rápidos, incluso en sistemas complejos que se encuentran en química y biología.

El desarrollo de cualquier material requiere su caracterización completa para encontrar posibles aplicaciones. En este caso se utiliza esta técnica para dilucidar la estructura molecular destacando en aplicaciones las fibras de coco como material de embalaje. De hecho, esta técnica parece ser uno de los métodos más interesantes utilizados por muchos investigadores para evaluar con precisión la composición de los materiales lignocelulósicos en términos de estructura de madera, celulosa y lignina. En este trabajo, se realizó un análisis del espectro infrarrojo de fibras de coco para investigar la estructura de ella, así como también proporcionar la base para seleccionar este tipo de fibras 
prometedoras que se utilizarán para posibles aplicaciones como materiales de embalaje.

Esta información podría ser utilizada para proponer aplicaciones prácticas para estas fibras, que puede tener los beneficios adicionales mencionados anteriormente y generar empleo junto con ventajas económicas para todos los países productores de coco del mundo. Como objetivo principal se tiene, determinar la composición química de compuestos orgánicos de las fibras de coco, utilizando espectroscopía de infrarrojo (IR), para caracterizar la fibra en sus principales bandas atribuidas a la presencia de celulosa, hemicelulosa y lignina.

\section{Materiales y métodos}

Se recolectaron desechos de puntos de venta de agua coco en el municipio de Texcoco de Mora, Estado de México, México, el origen de las frutas fue de las regiones de Acapulco y San Jerónimo de Juárez, del estado de Guerrero, México, se cosecharon en su punto de madurez apto para comercializar el agua. Posteriormente, se desfibraron manualmente y clasificaron.

El procedimiento para obtener el espectro de infrarrojo fue el siguiente:

Se realizó una selección de fibras de diferentes partes del coco con diferencias significativas en longitud y grosor, las diferentes muestras fueron caracterizadas utilizando su espectro de infrarrojo, los cuales se tomaron en un equipo IR Cary630 Agilent equipo disponible en el Laboratorio de Productos naturales de la Universidad Autónoma Chapingo.

Para el ensayo una muestra de fibras de coco se pulverizó en un mortero de porcelana, en esta ocasión no se utilizó una matriz de $\mathrm{KBr}$ (Bromuro de potasio). La toma de espectro se realizó en tipo sólido. Las fibras fueron analizadas midiendo los valores de absorbancia en el intervalo de $4000 \mathrm{~cm}-1$ a $400 \mathrm{~cm}-1$ del espectro electromagnético.

\section{Resultados y discusión}

Los espectros obtenidos en la región de infrarrojo permitieron observar las principales vibraciones, a las cuales se les asignan grupos funcionales, comparando con estudios ya realizados a otras fibras se determina cierta estructura para la fibra de coco.

En el espectro de IR de la fibra perteneciente a la región de Acapulco Guerrero (Figura 1) y San Jerónimo de Juárez (Figura 2), se identificó un pico de absorción en $3327.7 \mathrm{~cm}-1$ y $3320.9 \mathrm{~cm}^{-1}$ respectivamente, representando una deformación axial del enlace $\mathrm{OH}$ (hidroxilo) este pico es asignado a polisacáridos (celulosa, hemicelulosa y lignina) de la fibra de coco (Benini, 2011) y también se presta atención a una banda a $2890.9 \mathrm{~cm}^{-1}$ y 2881.8 $\mathrm{cm}^{1}$ respectivamente, presente en polisacáridos, esta vibración representa al grupo alifático $\mathrm{CH}$ conectado al anillo aromático y confirma la presencia de lignina (Silverstein, et al., 1994).

Se observó un pico muy grande e intenso en $1028.1 \mathrm{~cm}^{1}$ y $1023.9 \mathrm{~cm}^{1}$ para cada fibra, el cual se les atribuye a enlaces C-O de alcoholes y se relacionan con la molécula de celulosa (Guedes \& Mulinari, 2014). Se observó en $1509.6 \mathrm{~cm}^{1}$ y $1509 \mathrm{~cm}^{1}$ respectivamente, el pico que corresponde al enlace $\mathrm{C}=\mathrm{C}$ de la molécula de lignina (anillo aromático) correspondiente a la fibra de coco, este pico se encuentra más intensa que en la comparación con los otros espectros de otras fibras naturales como lo son los bagazos de caña, sisal y yute, las propiedades que se atribuyen a esta fibra es que la molécula de lignina le ayuda a comportarse como un material viscoelástico, el cual al ser sometido a una carga de compresión o una fuerza de impacto la región amorfa (lignina) de la fibra funciona como amortiguador y es muy resiliente de los fenómenos aplicados, característica deseable en los materiales de embalaje (Sengupta \& Basu, 2017). Otras propiedades que se le asignan aún mayor contenido de lignina son: Descomposición microbiana, que consiste en pruebas aceleradas de descomposición microbiana revelan que la fibra de coco retuvo el $60 \%$ de su resistencia original incluso después de 75 días, mientras que el yute y el sisal perdieron su fuerza en un período de 21 días. (Rosa, et al., 2010). La alta durabilidad de 
la fibra de coco puede atribuirse a su mayor menor en comparación con el yute y el sisal contenido de lignina y hemicelulosa y pectina mucho (Bhattacharyya \& Paul, 1980).

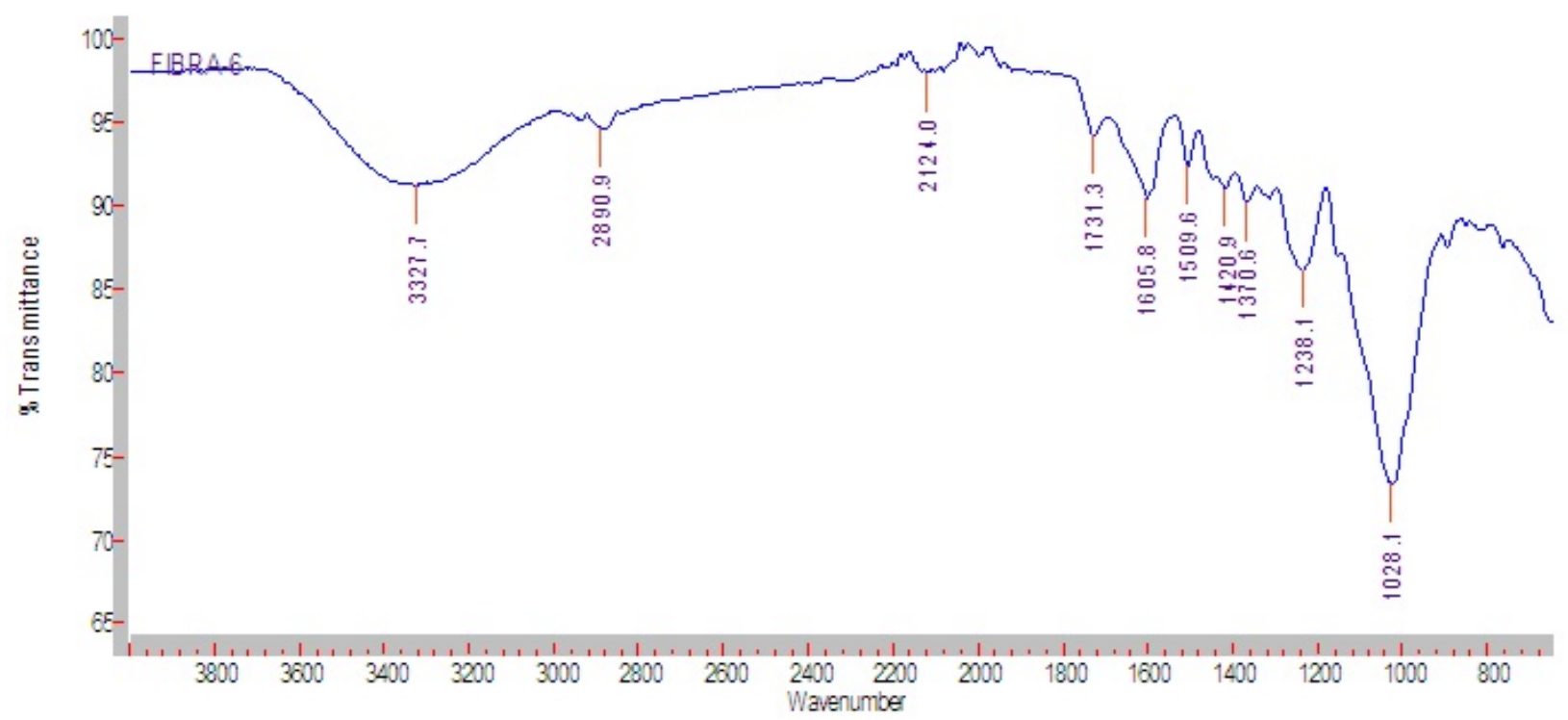

Figura 1. Espectro de IR de la fibra perteneciente a la región de Acapulco, Guerrero

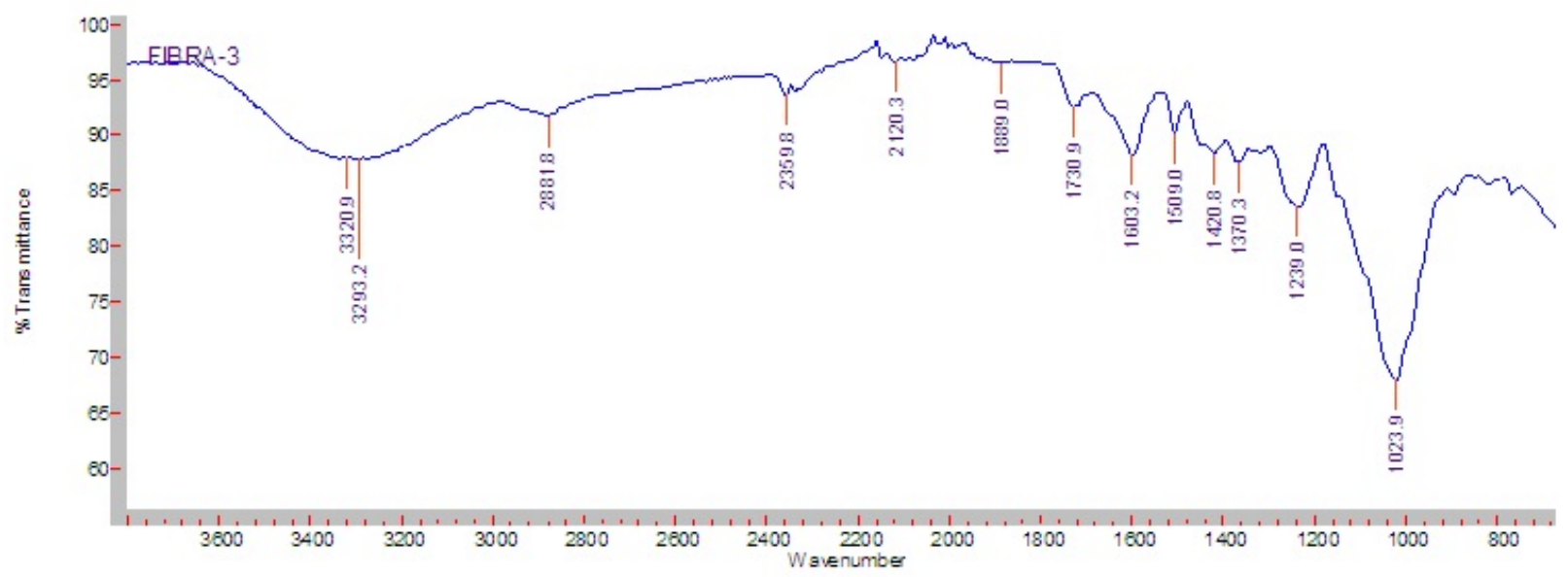

Figura 2. Espectro de IR de la fibra perteneciente al municipio de San Jerónimo de Juárez, Guerrero

\section{Conclusiones}

Se determinaron los compuestos orgánicos de la fibra de coco mediante la espectroscopía de infrarrojo con lo cual se observaron las principales bandas, concluyendo que la caracterización química revela la presencia y una alta cantidad de celulosa y lignina presente, lo cual puede orientar a que la fibra sea utilizada como material de relleno en embalajes.

\section{Referencias bibliográficas}

Benini, K., 2011. Desarrrollo y caracterización de compuestos poliméricos reforzados con fibras lignocelulósicas: fibra de la corteza de coco verde y bagazo de caña de azúcar, (tesis de maestría). Universidad Estatal Pualista, Guarantinguetá, Brasil. 
Bhattacharyya, S. K., Paul, N. B., from Renewable Resources. Berlín Heidelberg: 1980. Durability of long vegetable fibers. Springer.

IndianTextileJournal91,75-79. IndianTextileJournal, 91,75-79.

Rosa, M. F., Medeiros, E. S., Malmonge, J. A., Gregorski, K. S, Wood, D. F, Mattoso,

D'Lemos, A. L., Prestes-Pires, P. G., L. H. C, Glenn, G., Orts, W. J., Imam, Dealbuquerque, M. L., Botaro, V. R., Paiva, S. H., 2010. Cellulose nano-whiskers from J. M. F., Domingues-Junior, N. S., 2017. coconut husk fibres: Effect of preparation Biocomposites reinforced with natural fibers: conditions on their termal and morphological thermal morphological characterization. Materia. behavior. Carbohydrate Polymers, 81,83-92. DOI: $10.1590 /$ S1517-707620170002.0173

https://doi.org/10.1016/j.carbpol.2010.01.059

Guedes, R. I., Mulinari, D. R., 2014. "Caracterización mecánica de compuestos de PBED reforzados con fibras de palma. Cadunos Unifoa, Edición Especial del curso de maestría profesional en materiales

Sengupta, S., Basu, G., 2017. Properties of coconut fiber. Kolkata, India: Elsevier ICAR-National Institute of Research on Jute \& Allied Fibre Technology.

doi:10.1016/B978-0-12-803581-8.04122-9

Padilla, E. R. D., Pires, I. C. S. A., Yamaji, F. M., Fandiño, J. M. M., 2016. Producción y Caracterización Físico-Mecánica de Briquetas de Fibra de Coco y Paja de Caña de Azúcar. Revista Virtual de Química, 13. DOI: 10.21577/1984-6835.20160095

Silverstein, M. R., et al., 1994. Identificação espectrométrica de compostos orgânicos. Rio de Janeiro: Libros Técnicos y científicos.

Venkatachalam, N., et al., 2016. Effect of Pretreatment Methods on Properties of Natural Fiber Composites: A Review. Polymers \& Polymer

Reddy, N., Yang, Y., 2015. Innovative Biofibers Composites, Vol. 24, No. 7, 12.

La Revista Ingeniería y Región cuenta con la Licencia Creative Commons Atribución (BY), No Comercial (NC) y Compartir Igual (SA) 